\title{
MIMO Based Cognitive Radio Network with Efficient Spectrum Utilization
}

\author{
S. M. Patil, A. J. Patil
}

\begin{abstract}
This paper presents an energy-efficient power control scheme for Multi-Input-Multi-Output (MIMO) based multi-channel cognitive radio networks (CRNs) with adaptive mode selection protocol. Cognitive radio device operates in the temporal domain to utilize spectrum holes and MIMO system operates in the spatial domain. Thus CRN in collaboration with MIMO can produce a significant improvisation for spectrum utilization, and therefore, the integration of MIMO and CRN has received widespread research interest. To address the challenges of the collaboration of MIMO and CRN, we propose a MIMO based CRN network in this paper, in which basic CRN is modified with the QPSK transmission technique having four antenna elements per primary users (PU) and secondary users (SU). Similarly, MIMO significantly improves channel capacity particularly in SU transmission under low SNR condition, but issues like several antenna elements and specific antenna selection issues increase the computational complexity and energy consumption. The proposed network protocol strives to improve communication stability and rate of throughput via selecting the MIMO operating parameters such as multiplexing, diversity, or hybrid multiplexing-diversity gains. Specifically, we designed transmission power distribution along with an antenna selection scheme for enhancing the energy efficiency of CRN's under the influence of the maximum interference caused by the $S U$ to $P U$, utmost power transmission from $S U$, and the least rate of transmission through the SU link. Finally, the simulation results are compared with basic CRN alone using the BPSK scheme to evaluate and validate the proposed scheme.
\end{abstract}

Keywords: Multi-input-multi-output system, cognitive radio networks, primary users, secondary users and transmission power allocation.

\section{INTRODUCTION}

The last two decades have seen a drastic rise in the applications of wireless networks. In the wireless network service, enormous quantities of the spectrum are needed for their relevance. In the measurement of spectrum utilization, the Federal Communication Commission (FCC) reported that ineffective spectrum deployment occurs in the communication network, as the real spectrum utility ranges from 15 to $85 \%$ [1]. This spectrum utilization issue is resolved through the usage of novel adaptive spectrum admittance technology such as cognitive radio (CR) technology to efficiently occupy the accessible spectrum [2]. CR is an intelligent wireless communication technology that involved two kinds of consumers such as primary radio users (PU) and secondary users (SU).

Revised Manuscript Received on October 25, 2020.

* Correspondence Author

S. M. Patil*, Department of Electronics and Telecommunication Engineering Government College of Engineering, Jalgaon, Maharashtra, India

A. J. Patil, Shri Gulabrao Deokar College of Engineering, Jalgaon, Maharashtra-425002, India. E-mail:smpatil_entc@rediffmail.com

(C) The Authors. Published by Blue Eyes Intelligence Engineering and Sciences Publication (BEIESP). This is an open access article under the CC BY-NC-ND license (http://creativecommons.org/licenses/by-nc-nd/4.0/)
A PU also is known as a legacy user has the main concern to utilize the licensed bands, whereas SUs can exploit the underutilized licensed bands dynamically without disrupting the PUs operations.

The basic characteristics of CR are stated as: the cognitive ability that permits SUs to access and sense the informative message received from the working RF environment means recognizing idle channels, and cognitive reconfigurability that facilitates SUs to dynamically reprogram their working parameters (carrier frequency, bandwidth, power, modulation) in accordance with the interaction through RF environment [3]. In wireless communication systems, Multi-Input and Multi-Output (MIMO) technology has been adopted in past few years due to its potential to increase the quality of transmission and bandwidth utilization. In this technique, the transmitter and receiver are operational with several antennas ensuing in diverse propagation routes. In accordance with data transmission through given channel, MIMO technology can offer spatial diversity and/or spatial multiplexing gains. In contrast, $\mathrm{CRN}$ is also considered as a pledgeable service network to encourage the effectiveness in the spectrum utility for the wireless communication [4]. In the operation of CRN system, the SUs plays an significant role in the detection of spectrum holes, which means that spectrum bands that are not accessed by the PUs and subsequently utilized those vacant bands for their communication. As a result, the integration of MIMO and CRN is desired to enhance the transmission performance in the upcoming wireless communication services. Radio frequency RF chain is basically consisting of transmitters, receivers, threshold detector, amplifiers, attenuators, A/D converter, mixer, and so on [5]. In consequence, the several RF chains linked with numerous antennas at the transmitter and receiver side, which are energy rich due to the extend number of antennas and more transmission power. In order to resolve this network issue, selection of appropriate antenna can reduce the complication along with minimization of costing effect, but also acquire the benefits of MIMO technology, was discussed in. Energy efficiency in the communication network is defined as the number of transmitted message bits per unit energy between the nodes, which is the significant factor. So, extensive researches have been conducted in the development of energy efficient network model. Challenges in the energy efficient communication network model were studied with their solution in the CRN. In order to optimize energy efficiency of network model, the optimum transmission time period and power allocations with efficient energy have been examined [6][7].

\section{Published By:}

Blue Eyes Intelligence Engineering and Sciences Publication

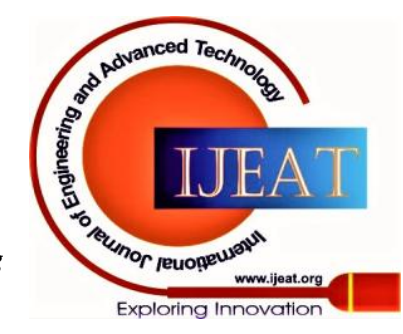


The common issue of power allocation and spectrum sharing in heterogeneous CRNs along with femtocells were studied in detail manner within the design of the energy efficient allocation scheme. In the modern research work, quality-of-service (QoS) of SU and their power constraints are consider to maximize the effective efficiency of SU.

Adaptive antenna selection methods can affect the energy efficiency, which did not concern in aforesaid research works. While more functional antennas in the MIMO channel can achieve better transmission rates, but require more power would also be expensive at the same time. Although more active antennas can achieve higher transmission rates in MIMO system, it will also cost more power at the same time. The problem of energy efficient power allocation in the MIMO channel system, along with the choice of antennas, was discussed in. Nevertheless, power allocation scheme still remains to be explored in the MIMO-based CRN with antenna selection technique [8-10]

To conquer the above network issues, in this paper we develop the energy-efficient scheme for a joint MIMO-based CRN system with an adaptive selection of antenna. The main objective is to maximize the energy efficiency via combined optimization over the power transmittance and the active antennas count under the restrictions of utmost interference from SU to PU. Besides, maximum power transmission from PU and the least amount of transmission rate in the SU link is considered. The simulation results of our paper show that the energy efficiency of MIMO based CRN are significantly improving due to the antenna selection technique. The rest of this paper is organized as follows. The MIMO based CRN system model is given in Sect. 2. In Sect. 3, proposed optimal energy efficient technique is described. The problem formulation and solution are given. Simulation results are presented and thoroughly discussed in Sect.4. Section 5 concludes this paper.

\section{MIMO-BASED CRN SYSTEM MODEL}

MIMO-based CRN System Model [6] as shown in Fig. 1, described in detail manner in this section. In the network model, SU share similar spectrum band along with PU, where transmitter and receiver of SU operated with Nt transmit and $\mathrm{Nr}$ receive antennas, respectively. It is assumed that the response of channel frequency HSS between transmitter and receiver module of SU is absolutely known to the SU receiver.

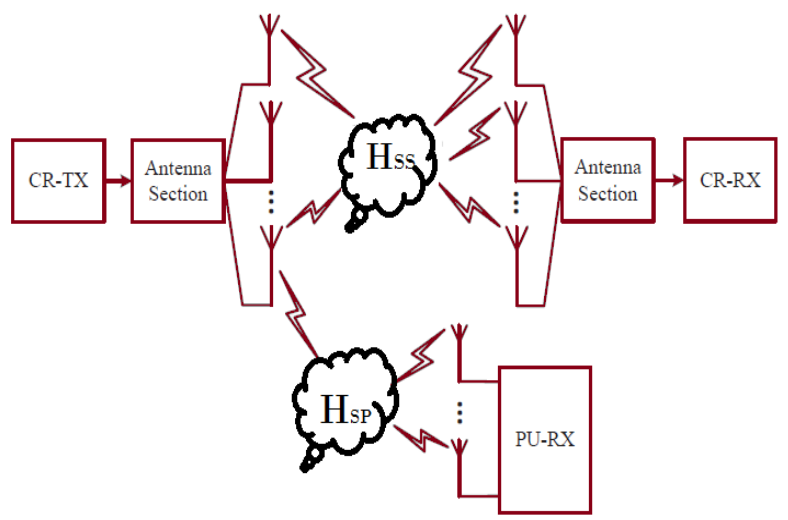

Fig. 1 MIMO-based cognitive radio networks with antenna selection.
Let us denote, transmit antenna of $\mathrm{SU}$ as $\Omega \mathrm{t}$ and receiver antenna of the selected SU as $\Omega$ r. As well the active numbers of transmit and receive antennas are represented as $\mathrm{Lt}=$ $\left|\Omega_{M}\right|$ and $\operatorname{Lr}=\left|\Omega_{L}\right|$. The instant frequency response of channel is indicated as Hsp is between the SU transmitter and the PU receiver. We consider that frequency response HSS and HSP are self-regulating and identically scattered Gaussian random variables, represented by $\mathrm{C}_{M}\left(0, \lambda_{1}\right)$ and $\mathrm{C}_{M_{M}}\left(0, \lambda_{2}\right)$, respectively. The PU and SU would interfere with one another in the spectrum sharing framework. The interference induced by the PU transmitter into the SU receiver can be designed as additive white Gaussian noise (AWGN) as per the central limit theorem (CLT) in a functional network with a sufficiently large number of sub-channels. The rate of transmission in the proposed network model is expressed as

$$
R=\sum_{n=1}^{\mathrm{Lt}} \log _{2}\left(1+\frac{P_{t}(n)\left\|H_{s S}\left[\Omega_{t}, \Omega_{r}\right]\right\|_{z}^{2}}{\delta^{2}}\right)
$$

Wherever, the subset of $H_{S S}$ is denoted as $H_{s s}\left[\Omega_{t}, \Omega_{r}\right]$ along with preferred transmit antenna subset given as $\Omega_{\mathrm{t}}$ and preferred receive antenna subset $\Omega_{\mathrm{r}}$ being utilized. Here, $\ell_{2}$ norm given as $\|\|,. P_{t}(n)$ is a power of transmission on the nth antenna.

With the intention that

$P_{t}=\left[P_{t}(1), P_{t}(2), P_{t}(3), \ldots \ldots P_{t}(N)\right]$ and noise in the network model is given as $\delta$. For feasibility of notation, we consider as:

$$
\mathrm{g}_{\mathrm{n}}=\frac{\left\|H_{s S}\left[\Omega_{\mathrm{t}}, \Omega_{r}\right]\right\|_{2}^{2}}{\delta^{2}}
$$

The energy efficiency of MIMO based CRN can be formulated as follows

$$
U=\frac{R}{\sigma \sum_{n=1}^{\mathrm{Lt}} P_{\mathrm{t}}(n)+P_{c}}
$$

The static circuit power is denoted as $P_{c}$ and the optimization dilemma exemplified via Eq. (3), can be derived as

$$
P 1: P_{t}, \Omega_{M}, \Omega_{N} \quad\left\{U=\frac{\sum_{m=1}^{L t} \log _{2}\left(P_{t}(n) g_{n}+1\right)}{a \sum_{n=1}^{L t} P_{t}(n)+P_{c}}\right\}
$$

Subject to,

$$
\begin{aligned}
& R \geq R^{*} \\
& P_{t} \geq P_{t}^{*} \\
& E\left\{\left|h_{s p}^{n}\right|^{2} P_{t}(n)\right\} \leq I_{t h}
\end{aligned}
$$

Here, the frequency response of channel $H_{S P}$ is given in the form of vector $\left[h_{S P y}^{1} h_{S P y}^{2} h_{S P}^{3} \ldots \ldots . . . h_{S P}^{n}\right]$, the constant parameter linked to the power amplifier efficiency is represented as $\alpha$, the minimum rate requirement in CR link is $R^{*}$, maximum power of transmittance from $\mathrm{SU}$ is $P_{t}^{*}$ and the maximum temperature threshold given as $I_{t h^{*}}$ The problem expressed in Eq.(4) could not have any practicable solution, when $\mathrm{R}$ could not achieve the subject along with their constraints mentioned in

Published By:

Blue Eyes Intelligence Engineering and Sciences Publication

(c) Copyright: All rights reserved.

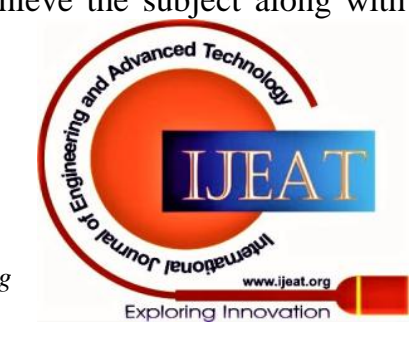


Eq.(6) and (7). In such case, the feasible solution can be obtained by decreasing minimum rate requirement through the SU. As per the chisquare distribution definition, the left side part of Eq.(7) can be given as

$$
E\left\{\left|h_{s p}{ }^{n}\right|^{2} P_{t}(n)\right\}=\lambda_{2} P_{t}(n)
$$

Consequently, Eq.(7) can be expressed as

$$
P_{t}(n) \leq I_{0}=\frac{I_{t h}}{\lambda_{n}}
$$

\section{OPTIMAL ENERGY EFFICIENT METHOD}

\subsection{Selection Process of Antenna}

The selection of antenna is the initial phase in the scheduling of optimal energy distribution issue. Antenna selection process carried out at the transmitter or receiver or at both sides. In this preference activity, we focus on the case where selection of antenna has been implemented at transmitter side along with the entire active transmit antennas. Furthermore, we can examine that the selection algorithms can also be applied at the receiver end in the similar mode [6][7].

Exhaustive Search Technique: For every Lt in the MIMO technology, we require to select the antenna subset along with the principal $\left\|\mathrm{H}_{\mathrm{ss}}\left[\Omega_{\mathrm{t}}, \mathrm{s}\right]\right\|_{2}$ which is represented as

$$
\Omega_{t}={ }_{1 \leq\left|\Omega_{t}\right| \leq \mathrm{N}_{t}}\left\|H_{s s}\left[\Omega_{t} ;\right]\right\|_{2}
$$

Wherein, $H_{s s}\left[\Omega_{t}, \Sigma\right]$ denotes subset channel matrix between subset of transmit antenna and all set of the receive antennas. The best solution can be determined through an extensive search over all the achievable subsets.

Antenna Selection: At one end, an exhaustive search carried out over all the antenna subsets. In this method, we needs to determine the antenna subset $\Omega_{t}$, which is related to the largest $\left\|H_{s s}\left[\Omega_{t},:\right]\right\|_{F}$, may be expressed as follows

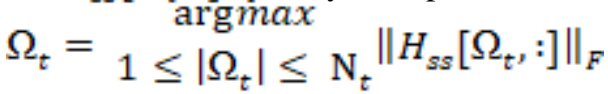

Whereas,

$$
\left\|H_{s s}\left[\Omega_{t} ;\right]\right\|_{F}=\sqrt{\sum_{l=1}^{N r} \sum_{k=1}^{L t} h_{l_{z} k}^{\Omega} h}
$$

Where, $h_{l_{2} k}^{\Omega}$ represent the element in the $\mathrm{l}^{\text {th }}$ row and $\mathrm{k}^{\text {th }}$ column of $H_{s s}\left[\Omega_{t}, \dot{ } ;\right.$ and $\|.\|_{F}$ indicate Frobenius norm.

3.2 Transmission Power Allocation with Constraints and Selected Antennas

Subsequent to the proper selection of antennas, transmission power allocation can also affect the optimal energy efficiency of network model. The main purpose of energy efficiency is to maximize transmit bits per unit energy [6]. In place of providing direct solution to the target problem in P1, Here we initiated two secondary optimization problems as follows:

$P 2: \underset{P_{t}(n), n \in \Omega_{t}}{\min }\left\{P_{t}\right\}$

Subject to:

$R \geq R^{*}$ and

$$
P_{t}(n) \leq \frac{I_{t h}}{\lambda_{2}}
$$

$$
P 3: \begin{gathered}
\min \\
P_{t}(n), n \in \Omega_{t}
\end{gathered}\{R\}
$$

Subject to:

$$
\begin{gathered}
P_{t} \geq P_{t}^{*} \\
P_{t}(n) \leq \frac{I_{t h}}{\lambda_{2}}
\end{gathered}
$$

Wherein, problem of optimization P2 is the minimization of transmit power and another optimization problem P3 is a transmission rate maximization issue. Without loss of generality, presume that $g_{1} \geq g_{1} \geq \cdots \geq g_{L t}$. The solution of power allocation for optimization issue P2 can be expressed as

$$
P_{t}(n)=\left[\mu-\frac{1}{g_{n}}\right]_{0}^{I_{0}}
$$

Where, $\mu$ is the level, for considering all values $\in \Omega_{t^{\prime}}$ $[x]_{a}^{b}=\min \{b, \max \{x, a\}\}$. The lower $\mu_{L}$ and upper bound $\mu_{U}$ of the feasible level region are the proper solution related to the problem of optimization $\mathrm{P} 2$ and $\mathrm{P} 3$ respectively. Therefore, an equivalent optimization problem P1 can be given as

$$
\begin{aligned}
& \text { P4: } \max _{\mu}\{U(\mu)\} \\
& \text { Subject to: }
\end{aligned}
$$

$$
\begin{array}{r}
\mu_{L} \leq \mu \geq \mu_{u} \\
P_{t}(n) \leq I_{0}=\frac{I_{t h}}{\lambda_{2}}
\end{array}
$$

The best level $\boldsymbol{\mu}$ * can be determined through line search method. In this work, we select bisection search technique, which needed a expected number of iterations and it is also application for network model. Hence, we can prepare the optimal transmission power allocation algorithm. At first, we must to be determining the optimal transmission power allocation schedule that related to all possible set of MIMO channel matrix. The optimize solution according to the best possible choice of the subset channel matrix can be solved through Algorithm 1. Depends on the optimal power allocations in the given subset proper selection in Algorithm 2 , we can find the optimal energy efficiency and also calculate the related Lt. At last, we will select Lt, which can be maximize energy efficiency of network model. Consequently, in this paper we jointly optimize the power of transmittance, count of active antennas, and their subsets at the transmitter or receiver end to maximize the optimal energy efficiency under the influences of constraints for utmost interference from SU to PU, greatest transmission power, and least transmission rate over CRN system model.

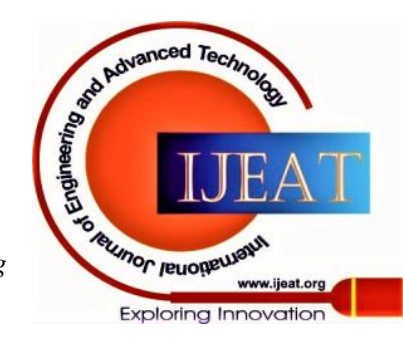




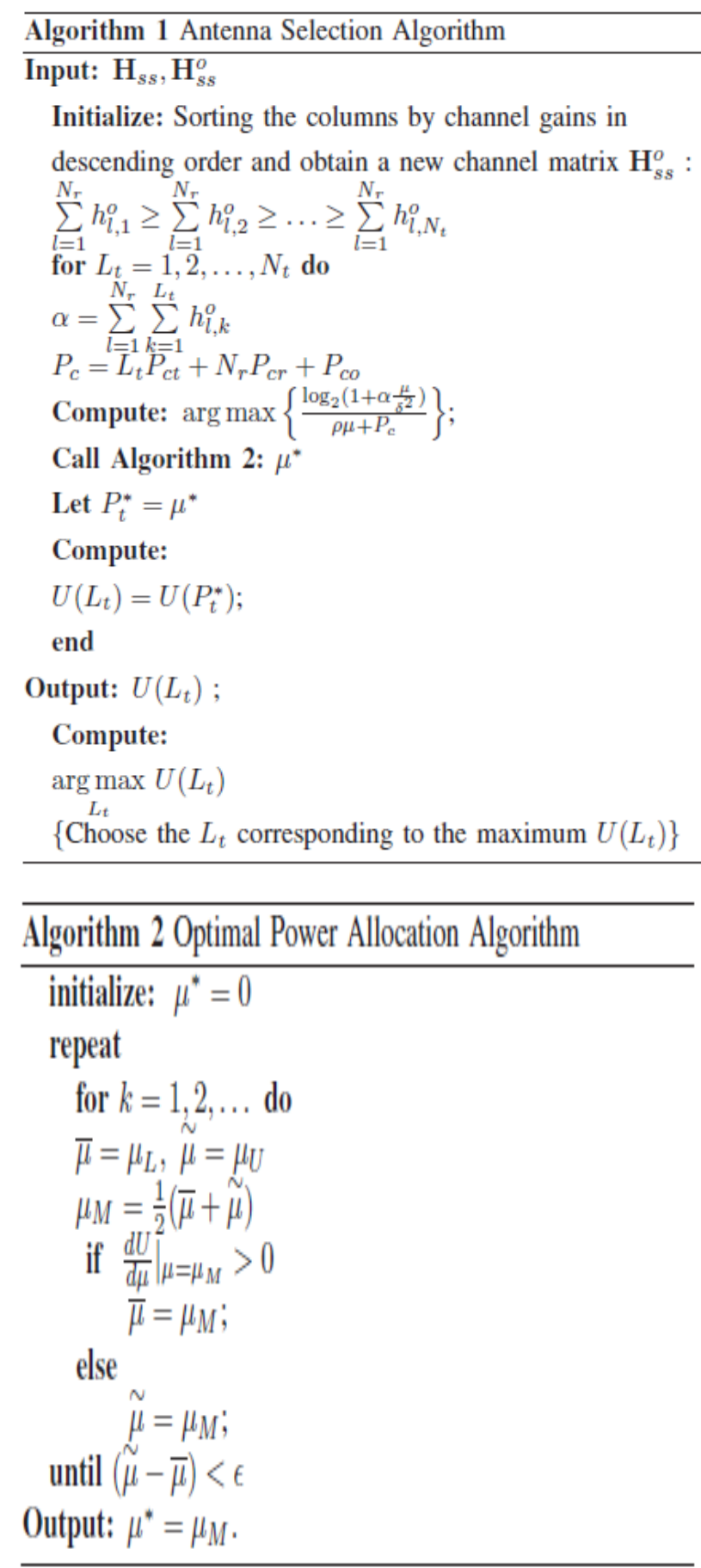

\section{SIMULATION RESULTS}

In this section, we performed experiments through simulations and use of MIMO system based CRN with optimal energy efficient technique to show the optimize performance through Algorithm 1 and 2. The obtained simulation results verify network model analysis and to exhibits the effectiveness of our proposed scheme. Simulations are conducted under the condition of averaging over 500 channel realizations. As per user requirement, we set parameter channel gain ratio in the MATLAB simulation as $\lambda$ $=\lambda 1 / \lambda 2$ and also presume the uniformly distributed distance between the transmitter and receiver node in CRN.

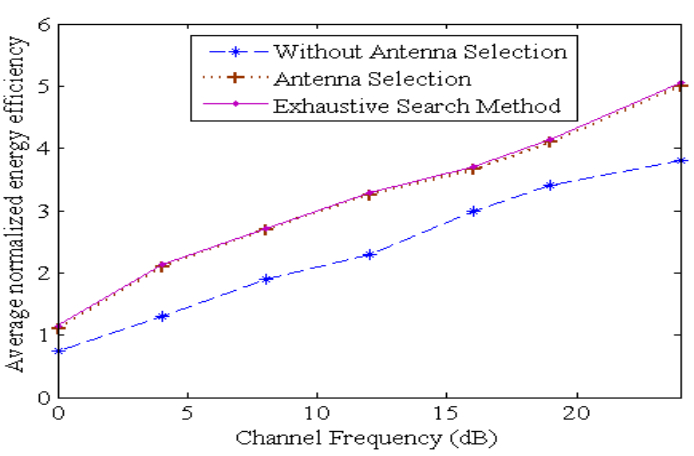

Fig. 2. Energy efficiency of network model versus different scheduling schemes

Fig. 2 shows the average value of normalized energy efficiency underneath various scheduling, in the simulation network parameters are chosen as $\mathrm{Nr}=4, \mathrm{Nt}=4$ and $\delta 2=$ $0.01 \mathrm{~W}$. Antenna selection algorithm tested at the transmitter side. It is clearly notice that the optimal energy efficiency can be enhanced through antenna selection technique. The antenna sequencing algorithm is able to attain the optimal solution underneath proposed MIMO-based CRN.

Since the channel gain parameter increases, then energy efficiency of network model also increases at the identical time, owing to the boosted received power and the adaptive selections of active antennas. As a result, evaluated with traditional techniques without utilizing antenna selection algorithm, the adaptive antenna selection based technique can attain important performance gain. In addition, in the low $\lambda$ region, the power transmittance dominates the overall power consumption, whereas, for high $\lambda$ region, the power transmittance in circuit plays vital role for the energy efficient design for proposed network.

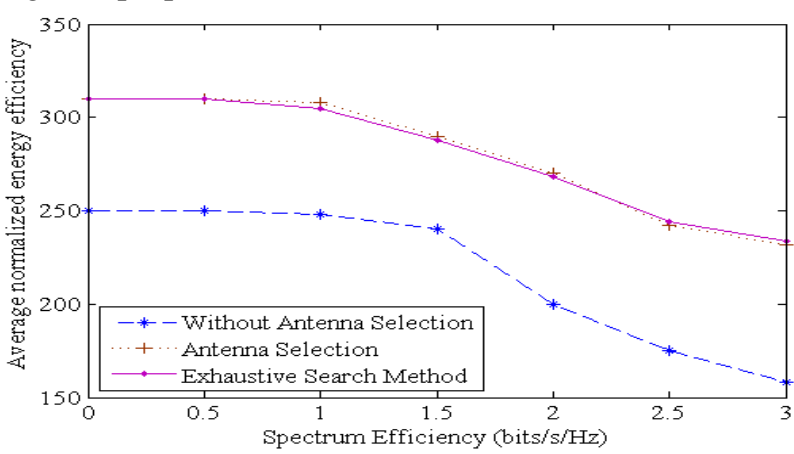

Fig. 3 Energy efficiency versus minimum spectrum efficiency (SE) requirement

Fig. 3 shows that the energy efficiency going to reduces with the raises of the least spectrum efficiency necessity. Since, this is the higher requirement of the least spectrum efficiency is, the superior transmitted power is required in both scheduling methods, which lead to the improve the energy efficiency. As a final point, it is observed that presented antenna selection based technique still perform well compared with the method that does not utilize antenna selection algorithm. Simulation results show that adaptive antenna selection method can enhance the energy efficiency in MIMO-based CRN.

Published By:

Blue Eyes Intelligence Engineering and Sciences Publication

(c) Copyright: All rights reserved.

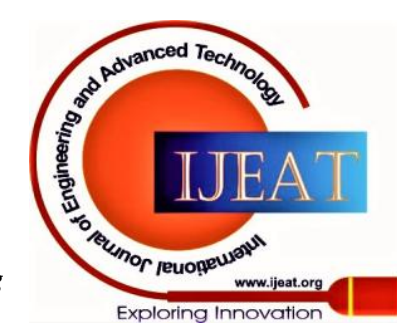




\section{CONCLUSION}

In this paper, we have analyzed the problem of effective spectrum utilization and their solution with energy efficient transmission power allocation in MIMO-based CRNs. Particularly; we proposed a joint adaptive mode selection and channel assignment with optimize performance for MIMO-based CRNs under the restrictions of the utmost interference to PU receiver, greatest transmission power allocation and least rate requirement in this simulation work. With considering increasing cost of RF chains in MIMO technology, here we employed the antenna selection method in proposed network model. In order to solve the problem of optimal energy efficiency, we applied proper antenna selection process with desired channel gain parameter. Then after, utilize transmission power allocation algorithm by using the bisection search technique. We have carried out simulation tests to confirm the efficacy of the proposed network model with energy efficient scheme under changing channel conditions and network parameters. The simulation results of proposed method shows the superior energy efficiency performance over traditional scheme for presented network model.

\section{ACKNOWLEDGMENT}

This research work is supported by TEQIP-III Institute: Government College of Engineerting, Jalgaon. The authors would like to express their sincere gratitude to the Editor-in chief and anonymous reviewers whose constructive comments have helped us to significantly improve both the technical quality and presentation of this paper.

\section{REFERENCES}

1. G. O. Young, "Synthetic structure of industrial plastics (Book style with paper title and editor)," in Plastics, 2nd ed. vol. 3, J. Peters, Ed. New York: McGraw-Hill, 1964, pp. 15-64.

2. Vibhuti Rana and P. S. Mundra, "A Review on QOS Parameters in Cognitive Radio Using Optimization Techniques", International Journal of Engineering and Innovative Technology (IJEIT), Vol 5(12), pp 59-63, 2016.

3. Van Tam Nguyen, Frederic Villain, and Yann Le Guillou, "Cognitive Radio RF: Overview and Challenges", VLSI Design, Hindawi Publishing Corporation, Volume 2012, Article ID 716476, 13 pages, 2012.

4. C. H. Uma, P. Dhana Lakshmi, N. Venkateswarao, "Blind Spectrum Sensing in Cognitive Radio using BPSK and QPSK Modulation Techniques", International Journal of Electronics, Electrical and Computational System (IJEECS), vol 6(6), pp 197-201, 2017.

5. Nikolaos I. Miridakis, Theodoros A. Tsiftsis and George C. Alexandropoulos, "MIMO Underlay Cognitive Radio: Optimized Power Allocation, Effective Number of Transmit Antennas and Harvest-Transmit Tradeoff', IEEE journal, Computer Science Information theory, arXiv:1801.07209v1 [cs.IT] 22 Jan 2018, pp 1-32.

6. V.Bindusri, M.Vijaya Lakshmi, "Power and Channel Allocation on MIMO Systems in Cognitive Radio Network", International Journal of Engineering Science and Computing (IJESC), Vol. 6(8), pp 2733-2738, 2016.

7. Shan Jin and $\mathrm{Xi}$ Zhang, "Optimal Energy Efficient Scheme for MIMO-Based Cognitive Radio Networks with Antenna Selection", IEEE Conference on Communication Network, Feb - 2015, pp 1-6.

8. G. Susmitha, V. Thrimurthulu, K. Jagadish kumar, "An analytical performance for multi-antenna Cognitive Radio Network (CRN) Systems", Anveshana's International Journal of Research in Engineering and Applied Sciences, (AIJREAS), Vol. 1(8), pp 43-53, 2016..

9. Gesualdo Scutari, Daniel P. Palomar, and Sergio Barbarossa, "Cognitive MIMO Radio - Competitive optimality Design Based on Subspace
Projections", IEEE Signal processing Magazine, Vol. 22(3), pp 46-59, 2008.

10. Sha Hua, Hang Liu, Mingquan Wu, and Shivendra S. Panwar, "Exploiting MIMO Antennas in Cooperative Cognitive Radio Networks", Research report by New York State Center for Advanced Technology in Telecommunications (CATT) and the Wireless Internet Center for Advanced Technology (WICAT), an NSF Industry University Cooperation Research Center, Feb - 2016, pp 1 - 9.

11. Suman Rathi, Rajeshwar Lal Dua, Parmender Singh, "Spectrum Sensing in Cognitive Radio using MIMO Technique", International Journal of Soft Computing and Engineering (IJSCE), Vol. 4(5), pp 259 - 265, 2011.

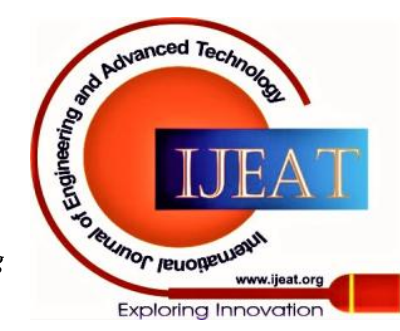

